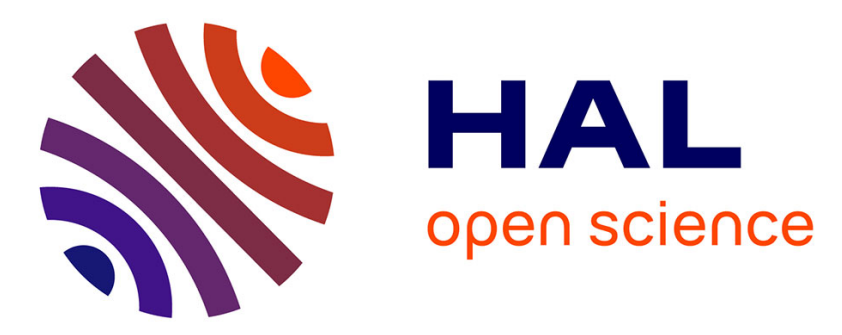

\title{
Le Drive, service complémentaire ou cannibalisation de l'offre des services des GMS : une analyse par le mix marketing et le mix logistique
}

Olivier Mevel, Thierry Morvan, Nélida Morvan, Magali Jara, Dany Vyt

\section{- To cite this version:}

Olivier Mevel, Thierry Morvan, Nélida Morvan, Magali Jara, Dany Vyt. Le Drive, service complémentaire ou cannibalisation de l'offre des services des GMS: une analyse par le mix marketing et le mix logistique. Logistique \& Management, 2017, Logistique et RSE, 25 (2), pp.119-130. 10.1080/12507970.2017.1336950 . halshs-01806408

\section{HAL Id: halshs-01806408 \\ https://shs.hal.science/halshs-01806408}

Submitted on 3 Sep 2018

HAL is a multi-disciplinary open access archive for the deposit and dissemination of scientific research documents, whether they are published or not. The documents may come from teaching and research institutions in France or abroad, or from public or private research centers.
L'archive ouverte pluridisciplinaire HAL, est destinée au dépôt et à la diffusion de documents scientifiques de niveau recherche, publiés ou non, émanant des établissements d'enseignement et de recherche français ou étrangers, des laboratoires publics ou privés. 


\section{Le Drive, service complémentaire ou cannibalisation de l'offre des services des GMS : Une analyse par le mix marketing et le mix logistique}

\author{
Olivier Mevel \\ Maître de Conférences \\ Université de Brest/LEGO \\ IUT de Brest \\ Olivier.mevel@univ-brest.fr
}

\author{
Thierry Morvan \\ Maître de Conférences \\ Université de Rennes 1/LEGO \\ IUT de Saint Malo \\ Thierry.morvan@univ- \\ $\underline{\text { rennes1.fr }}$
}

\author{
Nélida Morvan \\ Maître de Conférences \\ Université de Rennes 1 \\ IUT de Saint Malo \\ nelida.morvan@univ- \\ rennes1.fr
}

\author{
Magali Jara \\ Université de Nantes/LEMNA \\ IUT de Saint Nazaire \\ magali.jara@univ-nantes.fr
}

\author{
Dany Vyt \\ Université deRennes1/CREM \\ Institut de Gestion de Rennes \\ dany.vyt@univ-rennes1.fr
}

Résumé : L'objectif de cet article est d'étudier l'impact d'une nouvelle formule de service, le Drive, auprès du consommateur. Plus précisément, nous avons souhaité déterminer les variables créatrices de valeur permettant de prédire le comportement de recommandation du client. Nous avons mené une enquête empirique auprès d'un territoire spécifique: la Bretagne. Au regard des résultats, nous remarquons en particulier l'importance de la satisfaction du point de retrait comme facteur clé de succès

Mots clés : Drive, boucle de service, mix-marketing/mix-logistique, frontière coût/service

Drive-in service, a complimentary service or a cannibalization of retailers' service offering. An analysis from the marketing mix and from the logistics mix

Summary: This article aims to study drive-in users' experiences. It focuses on identifying key variables. These variables generate values which enable the prediction of the customer's recommendation behavior. We have carried out an empirical study in Brittany. Considering the results, we note the importance of the point of withdrawal satisfaction rate as a key factor of success.

Keywords: Drive, service loop concept, marketing mix/logistics mix, frontier cost/service 


\section{Introduction}

Douze ans après la première ouverture, la France compte plus de 3800 Drives alimentaires ${ }^{1}$ en 2015. Le Drive s'est donc imposé comme une suite naturelle à l'évolution des premières formules de services (supermarchés, hypermarchés, proximité...) liées à la vente de produits alimentaires en répondant aux impératifs sociétaux du consommateur pressé et précautionneux de ses dépenses. Véritable gisement de croissance ${ }^{2}$ interne pour les enseignes de distribution qui ont su y investir, ce nouveau circuit peut désormais représenter jusqu'à $60 \%$ de la croissance endogène d'une grande surface alimentaire. Dès lors, l'ensemble des enseignes tentent de se repositionner du point de vue de la personnalisation de la relation de service qu'elles entretiennent avec leurs clients. Le Drive participe ainsi d'un renouveau des aspects économiques et transactionnels de la relation de service (Lovelock, 2011) établie par les Grandes Surfaces Alimentaires (GSA) avec le client.

Il est important de souligner que trois modèles dominants de service au volant coexistent et différent selon la stratégie envisagée par le distributeur : le Drive déporté ou isolé (Drive pure player), le Drive accolé ainsi que le service Drive en picking. Cependant, quelle que soit la complexité de la formule de service mise en œuvre, ce nouveau canal de distribution permet aux réseaux logistiques de lever le problème du dernier kilomètre (Colla et Lapoule, 2011 ; Picot et alii, 2009 ; Punakivi et alii., 2001) tout en permettant un redéploiement et une simplification de la couverture territoriale au sein d'une même zone de chalandise. Enfin, elle répond aux exigences actuelles du shopper: se libérer des achats corvée en magasin physique (Colla, et Lapoule, 2015 ; Huré et Cliquet, 2011) sous contrainte de disponibilité des produits tout en optimisant la gestion de son temps d'achat.

Ainsi, la formule de service «Drive » des distributeurs est adossée à des propriétés marketing et logistiques remarquables car relativement dissemblables des premières formules de service développées par les distributeurs jusqu'alors intégralement basées sur l'exploitation intensive d'une surface commerciale dans une zone de chalandise. En effet, en Drive, la maximisation du taux de marge de l'enseigne va très directement dépendre d'un taux de service client construit, à la fois, en front-office, sur la pertinence d'un mix-marketing numérique (image de l'enseigne, assortiment, prix) mais aussi sur la minimisation, en back-office, des coûtssystèmes issus de la gestion du mix-logistique (coût de traitement des commandes, coût de transport, coût intrinsèque des stocks, coût d'entreposage, coût de préparation des commandes

\footnotetext{
${ }^{1}$ Source : Kantar, décembre 2015.

${ }^{2}$ Respectivement $50 \%$ et $60 \%$ de la croissance endogène du Groupement E.Leclerc sont expliqués par le Drive en 2013 et 2014
} 
client). Par conséquent, tout l'enjeu du déploiement d'un management opérationnel des services pertinent en Drive est de bien comprendre le niveau de service exigé par le client et donc de déterminer les variables créatrices de valeur du Drive susceptible de prédire le comportement de recommandation du client.

Par conséquent, au travers de nos recherches, nous avons souhaité étudier, à la fois, d'un point de vue physique et numérique, l'impact de cette nouvelle forme de service auprès du consommateur.

Pour répondre à notre problématique, dans un premier développement (1), nous nous attachons à comprendre les spécificités liées à la mise en œuvre de la formule de service «Drive » en tant que nouvelle formule de service spécifique à la GMS. Dans un second temps (2), nous avons souhaité mener une étude empirique quantitative afin d'étudier le comportement de recommandation de l'utilisateur du Drive du point de vue de la servuction produite par l'enseigne, à la fois, d'un point de vue digital et numérique mais aussi d'un point de vue physique.

\section{1- Drive relation de service et géomarketing}

Nous nous intéresserons successivement aux caractéristiques liées à la mise en œuvre de la formule Drive (1.1) avant de présenter une analyse de ce même Drive en termes de mixmarketing et de mix-logistique (1.2) puis, dans un dernier point, nous évoquerons les contraintes géomarketing qui pèsent sur le Drive (1.3).

\section{1-1- Le Drive : un complément aux différentes formes de service proposées par le distributeur}

Depuis les années 50, dans l'ensemble des pays industrialisés, les GSA ont développé une relation de service essentiellement basée sur la mise en œuvre de services standardisés de masse à faible intensité de relation avec le personnel de contact (Baranger et alii, 2009). Cette mise en œuvre de services, autrement aussi appelée servuction (Eiglier et langeard, 1987), a été progressivement déclinée par les GSA autour des six principales formules de services de distribution que sont l'hypermarché, le supermarché, le hard-discount, la proximité, le Drive et la livraison à domicile. Depuis 60 ans, la grande distribution alimentaire a donc su évoluer d'une simple logique produit vers la gestion d'un couple homogène produit/service qui 
s’inscrit désormais dans un nouveau modèle stratégique des services déjà initié par les Apple, Google, Amazon et autres Facebook et Twitter. En effet, en dépit des apparences, les stratégies des géants américains du Web sont similaires à celles de la grande distribution mondiale (Walmart, Tesco, Wholefood,) à savoir parvenir à enfermer l'utilisateur dans un univers d'offres complémentaires pour mieux se rémunérer au final sur les services qui lui seront fournis.

Dans le secteur des services, depuis 2007 et le tournant lié au lancement de l'IPhone notamment, si le GAFAT (Google, Apple, Facebook...) s'est mieux différencié que des entreprises aussi puissantes que HP, IBM, Oracle, Intel, Nokia, Motorola, RIM, c'est parce qu'ils ont proposé un multitude de modes d'accès au service. C'est un renversement complet de perspective pour les plus grands retailers mondiaux qui comprennent soudainement que, d'une part, la concurrence et les plus grands dangers viendront désormais des entreprises issues d'un univers numérique comme Amazon et que, d'autre part, puisque l'intelligence est désormais dans le réseau, il faut y avoir rapidement accès (en réponse à la connectivité mobile du consommateur). Par conséquent, dans cet univers, le logiciel est désormais la clé de gestion de services devenus plus complexes. C'est dans ce contexte qu'est né le Drive qui, au sens du triangle des services de Gadrey (1992), reste une formule de service spécifique aux prestataires GSA se traduisant par la mise à disposition d'un potentiel technique support plus ou moins automatisé (site Internet, système d'information, entrepôt) à destination d'un consommateur qui lui reconnait une réelle valeur d'usage.

Nous entrons dans une période de révolution des services dont l'enjeu est clairement le contrôle des usages grand public et cela les grands distributeurs mondiaux l'ont parfaitement compris..

Dès lors, face à l'évolution de la hiérarchisation des critères de choix du consommateur en faveur de l'économie digitale ${ }^{3}$, la grande distribution alimentaire (GMS) n'a eu d'autres choix que d'évoluer progressivement vers des stratégies de bi-canalité puis de multi-canalité voire d'omni-canalité aujourd'hui. Par ailleurs, si les enseignes investissent autant sur le Drive actuellement, c'est aussi, sans doute, parce que la question du juste format des magasins leur est clairement posée par un client dont les cinq premiers critères de choix sont devenus en 2016 : le sentiment de faire une bonne affaire, le gain de temps, la disponibilité du produit, l'expérience client et l'obtention d'une gratification liée à la fidélité (Mevel et alii, 2016).

\footnotetext{
${ }^{3}$ Au Royaume-Uni, 20\% du commerce de détail a eu lieu sur le Web en 2015 (Financial Times)
} 
Dans un contexte déflationniste où les distributeurs s'affrontent actuellement au travers d'une guerre des prix fratricide, le multicanal a donc le vent en poupe et cela pousse clairement à un développement continu des Drive ( $c f$. figure 1).

Figure $n^{\circ} 1$ : l'explosion du nombre de Drive dans la grande distribution alimentaire

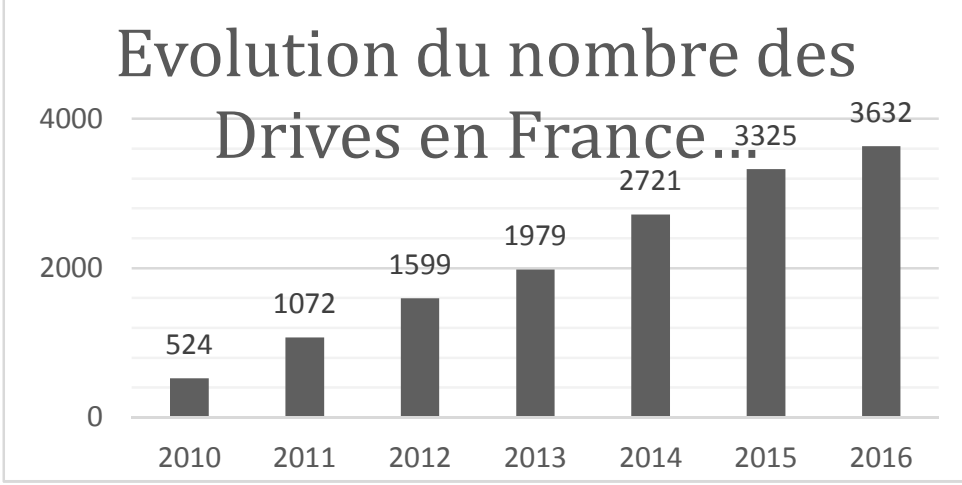

Il n'est donc pas étonnant de constater que la nouvelle formule de service Drive se caractérise essentiellement au travers de deux éléments fondamentaux que sont le design et le logiciel : Le design du site internet en tant qu'interface créative du parcours client en front-office et le logiciel comme contenu essentiel à la mobilisation et à la synchronisation de bases de données clients, de disponibilité des produits, de coordination de la gestion et de la préparation des commandes. En ce sens, le Drive peut être défini comme un processus digital et physique de distribution alimentaire, sans existence autonome, mettant face à face un client et une enseigne durant une phase de commande, de production et de consommation du service par le client que l'on peut qualifier de relation de service (Mevel et Morvan, 2015).

Le Drive alimentaire est donc une formule de service propre au distributeur dont le rôle va être de fabriquer le service au moment et à l'endroit où une demande existe. Plus précisément, la délivrance du service Drive au consommateur implique, au sens de la théorie (Kurz et Clow,1998 ; Lovelock et Lapert,1999 ; Volle, 2000), une co-construction de la valeur entre le consommateur et le distributeur.

Les caractéristiques du Drive, en termes de fabrication du service, prennent racine dans la servuction, qui est l'organisation systématique et cohérente de tous les éléments physiques et humains nécessaires à la réalisation d'une prestation de service, dont le niveau de qualité a été préalablement déterminé. Cela signifie aussi que le taux de service d'un Drive dépend positivement du parcours client étudié et développé dans une stratégie de Customer Experience Management (CEM) qui seule permet de rendre compte de la qualité perçue par le 
client. Ainsi, le parcours client apparait comme la ligne maîtresse de la servuction en Drive. Ce parcours client qui doit être très précisément décrite dans un document référence, le blueprint ou logigramme de la valeur. L'élaboration d'un tel document permet d'illustrer les différentes dimensions du temps, de visualiser les points d'interactions, d'identifier et de corriger les points d'étranglement et de redondance dans le but d'optimiser la prestation de service voire de permettre le développement de nouveaux services.

En définitive, dans le cas de la formule de service «Drive» proposée, le couple produit/service est profondément imbriqué et le client, qui achète rarement un produit sans tenir compte des services qui l'accompagnent, attend aujourd'hui de son enseigne de distribution un ensemble de fonctions servicielles clés. Le distributeur est donc conduit à développer de plus en plus de services complémentaires associés en Drive tout en mesurant au plus près son taux de service relativement à ses promesses marketing et aux attentes suscitées chez ses clients. De la juste adéquation entre le mix-marketing et le mix logistique dépend le taux de service final reconnu par le client de l'enseigne à la mise en ouvre de sa formule Drive.

\section{1-2- Le Drive : une analyse par le Mix Logistique/Mix marketing}

Le Drive est une formule de service dont le front office est numérique et interactif, impliquant un échange permanent entre le site internet de l'enseigne et le client pendant la phase de commande. Le front office d'un Drive est complexe car il exige une synchronisation du merchandising de l'assortiment avec essentiellement en back office la logistique dont l'objectif est de délivrer la commande lors du retrait sous conditions de l'accessibilité ; cette synchronisation devant assurer la qualité de service recherché par le client. Plus précisément, le taux de service est construit à la fois en front office sur la pertinence d'un mix-marketing numérique (image de l'enseigne, assortiment, prix) et sur la minimisation, en back-office, des coûts issus de la gestion du mix-logistique. Cette gestion consiste, pour un niveau donné de service clientèle, à minimiser le coût logistique total. Il se définit comme la somme de cinq principaux coûts logistiques (coût de traitement des commandes, coût de transport, coût intrinsèque des stocks, coût d'entreposage, coût de préparation des commandes clients) qui ont la particularité de réagir de façon systémique (Samii, 2004 ; Lambert, 1994 et 1976, Shapiro et Heskett, 1985). Plus précisément, ce sont donc les cinq coûts logistiques qu'il va s'agir de traiter comme un ensemble car ils sont profondément reliés les uns aux autres : la 
minimisation de la somme de ces coûts étant potentiellement inférieure à la minimisation de chacun des cinq coûts pris indépendamment. Par ailleurs, la gestion du mix-logistique doit impérativement prendre en compte le niveau de service exigé par le client final en termes de disponibilité du produit au moment de la commande mais aussi de délai attendu pour réceptionner la commande numérique dans une fourchette horaire acceptable. Dès lors, l'arbitrage entre le mix-marketing numérique et le mix-logistique nécessite de bien comprendre l'interaction entre le niveau de service exigé par le client et les autres composantes de la stratégie marketing initiée par le distributeur.

Or, selon les stratégies envisagées par le distributeur, le modèle «Drive » choisi implique d'importantes spécificités logistiques ( $c f$. tableau 1)

Tableau $n^{\circ} 1$ : Les spécificités logistiques des différents modèles de Drive

\begin{tabular}{|c|c|c|c|}
\hline & Drive Solo & Drive Accolé & $\begin{array}{l}\text { Drive Picking magasin } \\
\text { (store picking) }\end{array}$ \\
\hline Caractéristiques & $\begin{array}{l}\text { Le Drive est sur une autre } \\
\text { zone de chalandise }\end{array}$ & $\begin{array}{l}\text { Le Drive est sur le site du } \\
\text { même point de vente }\end{array}$ & $\begin{array}{l}\text { Le Drive dépend d'un } \\
\text { hypermarché voire d'un } \\
\text { super marché }\end{array}$ \\
\hline Stratégies envisagées & Stratégie offensive & Stratégie Défensive & Stratégie défensive \\
\hline Entrepôt & $\begin{array}{l}\text { Entrepôt de } 4000 \text { à } 5000 \\
\mathrm{~m}^{2} \\
\text { L'entrepôt est autonome } \\
\text { Situé aux abords des } \\
\text { grandes voies de } \\
\text { circulation }\end{array}$ & $\begin{array}{l}\text { Entrepôt de } 1500 \text { à } 2000 \\
\mathrm{~m}^{2} \\
\text { L'entrepôt est dédié mais } \\
\text { accolé à un magasin } \\
\text { traditionnel }\end{array}$ & $\begin{array}{l}\text { Un espace d'accueil de } \\
150 \text { à } 300 \mathrm{~m}^{2}\end{array}$ \\
\hline Logistique amont & Dédiée & $\begin{array}{l}\text { Mutualisée avec celle du } \\
\text { magasin voire dédiée }\end{array}$ & $\begin{array}{l}\text { Mutualisée avec celle du } \\
\text { magasin }\end{array}$ \\
\hline $\begin{array}{l}\text { Préparation des } \\
\text { commandes }\end{array}$ & $\begin{array}{l}\text { Préparation des } \\
\text { commandes dans un } \\
\text { entrepôt dédié } \\
\text { Temps de préparation de } \\
\text { commande : } 10 \text { minutes } \\
\text { en moyenne } \\
\text { Chemin de picking } \\
\text { optimisé par famille de } \\
\text { produits suivi d'un } \\
\text { regroupement par } \\
\text { commande client } \\
\text { Automatisation de la } \\
\text { préparation } \\
\text { Personnel dédié à la } \\
\text { préparation }\end{array}$ & $\begin{array}{l}\text { Préparation des } \\
\text { commandes dans un } \\
\text { entrepôt dédié } \\
\text { Temps de préparation de } \\
\text { commande : } 10 \text { minutes } \\
\text { en moyenne } \\
\text { Chemin de picking } \\
\text { optimisé par famille de } \\
\text { produits suivi d'un } \\
\text { regroupement par } \\
\text { commande client } \\
\text { Automatisation de la } \\
\text { préparation sauf parfois } \\
\text { pour les produits frais } \\
\text { pris en magasin } \\
\text { Personnel dédié à la } \\
\text { préparation }\end{array}$ & $\begin{array}{l}\text { Préparation des } \\
\text { commandes effectuée sur } \\
\text { la surface de vente } \\
\text { Temps de préparation de } \\
\text { commande entre } 20 \text { et } 50 \\
\text { minutes } \\
\text { Chemin de picking } \\
\text { dépendant des circuits du } \\
\text { magasin } \\
\text { Personnel polyvalent }\end{array}$ \\
\hline Gestion des stocks & $\begin{array}{l}6000 \text { à } 8000 \text { références } \\
\text { Gestion spécifique au } \\
\text { Drive } \\
\text { Ajustement journaliser } \\
\text { des stocks en fonction } \\
\text { des écarts constatés }\end{array}$ & $\begin{array}{l}6000 \text { à } 8000 \text { références } \\
\text { Gestion spécifique au } \\
\text { Drive sauf parfois pour } \\
\text { les produits frais pris en } \\
\text { magasin }\end{array}$ & $\begin{array}{l}\text { L'ensemble des } \\
\text { références en magasin } \\
\text { (entre } 20000 \text { et } 30000 \\
\text { références) } \\
\text { Gestion mutualisé avec le } \\
\text { magasin } \\
\text { Gestion de deux flux : } \\
\text { libre service et Drive }\end{array}$ \\
\hline
\end{tabular}


Plus précisément, au regard des trois types principaux de Drive, le Drive déporté ou isolé (Drive pure player), le Drive accolé ainsi que le service Drive en picking, deux modèles logistiques semblent émerger : la préparation des commandes en entrepôt et la préparation en magasin (store picking) (Rouquet, 2014 ; Durand, 2009, Baglin et al., 2005). Ainsi, le Drive solo repose sur la recherche permanente de l'optimisation de la préparation de commande; une optimisation nécessitant un volume important de commandes dans un environnement contraint, à la fois, par une limite physique (entrepôts entre 1500 et $6000 \mathrm{~m}^{2}$ avec 6 000/8 000 références proposées) et par le choix d'un mode de préparation au détail, à la différence des entrepôts habituels des distributeurs où les préparations s'effectuent au colis ou à la palette. $\mathrm{Au}$ contraire, le Drive picking en magasin, en s'appuyant sur un magasin de référence, est capable d'offrir un nombre de références bien plus important avec malgré tout un risque logistique en termes de rupture de stocks lorsque le nombre de commandes augmente.

Cependant, quel que soit la formule retenue, le défi logistique des distributeurs réside dans leur capacité à gérer des commandes dont le pic d'activité est souvent concentré sur un laps de temps très court ${ }^{4}$ pendant lequel l'organisation logistique du Drive doit être dans la capacité de gérer des flux de préparation de commande différents de façon simultanée avec des exigences en termes de service attendu: respect de la commande, choix de tranche horaire, temps d'attente, etc. En effet, comme le consommateur choisit la formule du Drive pour se débarrasser de tâches couteuses en termes de temps et d'effort, il va exiger que le distributeur, en charge de ces multiples tâches de manutention, soit dans la capacité d'organiser de telles opérations (Huré et al, 2013). Au final, la performance logistique doit faire partie intégrante de la transaction dans le cas d'une vente en ligne. Par contre, si la réception de la commande ne se réalise pas dans les conditions prévues (délais, erreurs de préparation récurrentes, etc.), le client reportera son choix sur un distributeur dont la performance logistique pourra le satisfaire. Pour autant, la performance d'un Drive semble rester aussi profondément dépendante des phénomènes liés à l'isochronie rencontrée par le consommateur au sein de sa zone de chalandise ce qui nous amène également à envisager une caractérisation du Drive autour du concept de géomarketing.

\section{1-3- Drive et géomarketing}

\footnotetext{
${ }^{44}$ Pour certaine enseignes $50 \%$ des commandes sont passés 2 à $3 \mathrm{~h}$ avant un retrait ; un retrait qui se concentre entre $17 \mathrm{~h} 30$ et $20 \mathrm{~h}$.
} 
L'intégration de critères spatiaux dans la politique marketing a donné naissance à une nouvelle discipline qui s'est positionnée à la croisée des chemins entre le marketing et la géographie : le géomarketing (Cliquet, 2002). Pour Douard (2002), il s'agit d' «une méthode de fédération et d'analyse des données basées sur leur dimension géographique. C'est une aide à la décision pour définir, mettre en cuvre et contrôler la stratégie commerciale dans le temps et l'espace. » D'une manière plus générale, le géomarketing ou marketing spatial, vise à intégrer l'espace et les données spatiales dans les décisions marketing (Cliquet, 2002, 2003). En d'autres termes, ici la question de recherche a trait au fait d'approcher voire de comprendre les logiques spatiales qui ont préfigurées à l'implantation mais aussi au développement des Drive en une décennie sur le territoire national.

Comme le rappellent Cliquet et alii. (2006), la mise en réseau du commerce de détail, ou réticulation, soulève de nombreuses réflexions en termes de gestion de l'espace territorial chez le distributeur. La prise en compte duale du critère géographique et du marketing spatial dans les stratégies des réseaux de distribution n'est pas nouvelle. Depuis longtemps, la localisation de chaque magasin s'avère décisive pour la rentabilité et la viabilité d'un réseau de distribution (Ghosh et Craig, 1983 ; Craig et alii., 1984), de même que le maillage du réseau et l'optimisation simultanée de sa réticularité au travers de la localisation des points de vente s'affirme comme une question prépondérante (Achabal et alii, 1982). Cette problématique répond à une logique d'expansion des réseaux et de croissance externe. Alors que l'implantation des nouveaux points de vente s'appuie sur de nombreuses stratégies spatiales éprouvées (Laulajainen, 1987 ; Laulajainen et Stafford, 1995), le Drive, quant à lui, ne bénéficie pour encore d'aucun soutien théorique.

De nombreuses questions demeurent alors. Quelles sont les conditions de succès d'implantation du Drive comme nouveau canal de distribution ? Dès 1931, Reilly établit une analogie entre la théorie Newtonienne de la gravité universelle et l'attraction commerciale des points de vente. Puis, pour intégrer davantage les comportements des consommateurs, de nouvelles techniques de définition de l'attractivité commerciale voient le jour. En 1962, Huff calcule la probabilité qu'un consommateur se rende dans un point de vente compte tenu du temps d'accès, d'une approximation de la distance, de la surface de vente ainsi que de l'ensemble de l'offre catégorielle présente dans la zone. Qu'en est-il réellement pour les Drive ? Peut-on modéliser leur attraction à partir des modèles traditionnels ou faut-il résolument développer d'autres formes de réflexion en ce qui les concernent ? Enfin, comment évaluer et modéliser la distance d'un mode de distribution vis-à-vis duquel le 
consommateur est par définition en mouvement entre son lieu de travail et son lieu d'habitation? La proximité joue-t-elle aussi un rôle dans la relation de service ? Dès lors, de quelle proximité parlons-nous ? L'absence de réponses à ces questions nous a amené à nous projeter sur le terrain afin de mieux comprendre les interactions en jeu.

\section{2- Aspects empiriques}

Après avoir rendu compte de la méthodologie quantitative de l'enquête, nous présentons nos résultats (2.2) pour enfin discuter des implications managériales (2.3)

\section{2-1- La méthodologie utilisée}

Afin, d'étudier le comportement de recommandation du Drive par le consommateur nous permettant de déterminer les variables créatrices de valeur, nous avons souhaité mener une enquête empirique auprès d'un territoire spécifique : la Bretagne. Le choix de la Bretagne s'explique par le fait qu'il s'agit d'une région motrice dans le développement des Drive en France et qu'elle apparait comme l'une des régions les plus denses avec plus d'un Drive pour 13000 habitants (contre 1 Drive pour 25000 habitants en moyenne) ${ }^{5}$.

Nous avons soumis un questionnaire auprès des utilisateurs du Drive. Le questionnaire construit comprenait trois parties principales pour répondre à notre problématique consistant à étudier d'un point de vue marketing et physique l'impact du Drive auprès du consommateur. La première partie de l'enquête consistait à étudier le comportement de l'utilisateur du Drive en termes de fréquentation, de catégories de produits commandés et de services attendus. Ensuite, il s'agissait de déterminer le degré de satisfaction du client dans son parcours numérique lui permettant d'élaborer sa commande mais aussi d'évaluer son degré de satisfaction en ce qui concerne la fonctionnalité et la pertinence du site internet (qualité des images, description des produits, etc.). Enfin, le questionnaire, dans sa dernière partie, avait pour objectif de déterminer le degré de satisfaction du client dans son parcours "physique », (accès routier, authentification, temps d'attente, relation avec le personnel du Drive).

A partir des données recueillies nous avons souhaité tester un modèle causal structurel (estimé par Path-PSL) afin d'expliquer le comportement de recommandation du Drive par cinq variables explicatives étudiées dans le questionnaire ( $c f$. annexe 1). Ces variables sont représentées par la satisfaction du point de retrait, du site internet, du service et des produits, ainsi que les freins (problèmes rencontrés dans le parcours numérique et le parcours

\footnotetext{
${ }^{5}$ Source : Top Drive mai 2014
} 
physique). Le modèle validé a pour objectif de déterminer les variables créatrices de valeur, autrement dit les facteurs clés du succès du Drive, par ordre d'importance. Ensuite afin de terminer les éventuelles différences avec le modèle général, nous l'avons testé sur les trois types de Drive existant : déporté, accolé et picking magasin.

Nous avons récolté les données en soumettant le questionnaire auprès d'un échantillon de convenance de 700 consommateurs des différents départements bretons ( $c f$. tableau n²). Les individus ont été interrogés en face-à-face à proximité des drives des enseignes qui ont donné leur autorisation.

Tableau n ${ }^{\circ} 2$ : Répartition de l'échantillon par département

\begin{tabular}{|c|c|}
\hline Départements & Pourcentage \\
\hline Côtes d'Armor & 28,5 \\
\hline Finistère & 31,1 \\
\hline Ille et Vilaine & 23,0 \\
\hline Morbihan & 17,4 \\
\hline Total & 100,0 \\
\hline
\end{tabular}

Le profil des utilisateurs du Drive de notre échantillon présentent de fortes similitudes avec celui d'études réalisées antérieurement : les clients des Drives sont à $95 \%$ des jeunes actifs, dont un plus des deux tiers ont entre 30 et 44 ans ; les deux CSP les plus représentées sont les employés $(37,1 \%)$ et les professions intermédiaires $(26,3 \%)$. Ils appartiennent à des foyers qui comptent en moyenne 3,4 personnes (contre 2,3 pour la moyenne des foyers français) ; les clients des Drives sont donc des parents (les $3 / 4$ des foyers comptent au moins 3 personnes) ( $c f$. annexe 4). L'enseigne principale du Drive fréquenté par les consommateurs de notre échantillon est dominée par Leclerc ( $c f$. annexe 5) et les Drive utilisés sont essentiellement les Drive déportés et accolés ( $c f$. annexe 5). Cette domination au sein de notre échantillon s'explique notamment par le fait que Leclerc est en position très favorable en ce qui concerne les Drive déportés tant en nombre de dépôts proposés que de nombre de pistes mis à disposition.

\section{2-2- Présentation des résultats}

Le premier résultat révélé par notre enquête souligne le fait que la recherche de proximité est une préoccupation majeure des utilisateurs d'une service tel que le drive. En effet, nous remarquons ( $c f$. annexe 2) que le critère «proximité de mon domicile » est celui qui domine l'ensemble des critères classés : 50,9\% des personnes interrogées le classent en première position. Ce résultat est renforcé par le fait qu'environ un individu sur deux accorde une 
certaine importance aux critères «Proximité de mon lieu de travail» $(48,1 \%$ des personnes interrogées ont classé ce critère) Par conséquent, la massification des achats de biens quotidiens, proposée par le Drive, offre la possibilité de réduire le temps contraint en créant une activité routinière et rationalisée.

Le second résultat est révélé par le modèle causal structurel testé et validé d'un point de vue statistique ( $c f$. annexe 1$)$.

Concernant les construits réflectifs largement utilisés en marketing, la procédure retenue suit celle proposée par Churchill portant sur l'examen de la fiabilité et de la validité convergente des construits latents. A l'issue de cette procédure, la validité discriminante est testée sur l'ensemble des construits latents du Comportement de recommandation du drive.

Les analyses confirmatoires ont été menées à l'aide du logiciel Xlstat PLSPM (basé sur la technologie PLEASURE ${ }^{6}$ ) fondé sur la modélisation Path-PLS.

Le Rhô de Jöreskog, est souvent utilisé (en complément de l'Alpha de Cronbach) pour tester la fiabilité des échelles de mesure (Chin, 1998). Pour l'ensemble des construits réflectifs, les indicateurs de validation de leurs mesures utilisés sont les suivants : les tests de fiabilité de l'échelle de mesure (la première valeur propre du bloc est supérieure à 1 et l'alpha de Cronbach et le Rhô de Dillon-Goldstein sont supérieurs à 0,7(Tenenhaus et alii., 2005); le test de la validité convergente (examen des communalités de chaque variable manifeste ${ }^{7}$ à sa variable latente (communalité intra) et examen de l'AVE (Average Variance Extracted) ou variance moyenne extraite, calculée sur la variable latente).

\footnotetext{
${ }^{6}$ Partial LEAst Squares strUctural Relationship Estimation. Ce logiciel a été développé par Chatelin Y.M., Esposito Vinzi V., Lauro C. et Tenenhaus M.

${ }^{7}$ Ou items de mesure.
} 
Tableau 4 : les indicateurs statistiques

\begin{tabular}{|c|c|c|c|c|c|c|}
\hline Variable latente & Dimensions & $\begin{array}{l}\text { Alpha de } \\
\text { Cronbach }\end{array}$ & $\begin{array}{l}\text { Rho de } \\
\text { D.G. } \\
\text { (ACP) }\end{array}$ & $\begin{array}{c}\text { Nombre de } \\
\text { conditionnement }\end{array}$ & $\begin{array}{l}\text { Valeur } \\
\text { critique }\end{array}$ & $\begin{array}{l}\text { Valeurs } \\
\text { propres }\end{array}$ \\
\hline SATISFACTION PRODUITS & 7 & 0.882 & 0.908 & 4.653 & 1.000 & $\begin{array}{l}4.114 \\
0.915 \\
0.512 \\
0.473 \\
0.433 \\
0.363 \\
0.190\end{array}$ \\
\hline $\begin{array}{l}\text { SATISFACTION SITE } \\
\text { INTERNET }\end{array}$ & 7 & 0.906 & 0.926 & 5.346 & 1.000 & $\begin{array}{l}4.511 \\
0.703 \\
0.654 \\
0.451 \\
0.355 \\
0.169 \\
0.158\end{array}$ \\
\hline FREINS & 1 & & & & & \\
\hline SATISFACTION SERVICE & 6 & 0.883 & 0.911 & 4.847 & 1.000 & $\begin{array}{l}3.792 \\
1.038 \\
0.526 \\
0.314 \\
0.169 \\
0.161\end{array}$ \\
\hline $\begin{array}{l}\text { SATISFACTION POINT DE } \\
\text { RETRAIT }\end{array}$ & 4 & 0.897 & 0.929 & 3.657 & 1.000 & $\begin{array}{l}3.059 \\
0.414 \\
0.298 \\
0.229\end{array}$ \\
\hline COMPORTEMENT & 1 & & & & & \\
\hline
\end{tabular}

Au regard des démarches statistiques précédentes, les échelles de mesure se confirment être chacune unidimensionnelle puisque seule la première valeur propre du bloc est supérieure à 1 et que l'alpha de Cronbach et le Rhô de Dillon-Goldstein sont tous deux supérieurs aux seuils recommandés. La fiabilité des échelles de mesure est par conséquent assurée.

La validité convergente des construits du modèle est également confirmée puisque les variances extraites dépassent les seuils recommandés ( $c f$. tableau ci-dessous).

Tableau 5 : Résultats de la validité convergente des construits du modèle

\begin{tabular}{|l|c|c|}
\hline \multicolumn{1}{|c|}{ Latent variables } & D.G. Rho & Average of variance extracted (0.644) \\
\hline SATISFACTION PRODUITS & 0.908 & 0.587 \\
\hline SATISFACTION SITE INTERNET & 0.926 & 0.644 \\
\hline SATISFACTION SERVICE & 0.911 & 0.631 \\
\hline SATISFACTION POINT DE RETRAIT & 0.929 & 0.765 \\
\hline FREINS & unidimensionnel & 1 \\
\hline COMPORTEMENT & unidimensionnel & 1 \\
\hline
\end{tabular}

Enfin, au regard de la matrice de validité discriminante ( $c f$. annexe 2), il semble que l'ensemble des construits du modèle mesure des dimensions à part entière. En effet, les 
variances partagées entre les variables latentes sont inférieures aux variances partagées (AVE en bas de chaque colonne) entre les variables latentes et leurs items de mesure respectifs.

Valider $^{8}$ le modèle structurel revient à tester la qualité des relations causales entre les six variables latentes du modèle et à identifier le poids ${ }^{9}$ de chacune des cinq variables explicatives dans la formation de ce dernier. Pour ce faire, plusieurs indicateurs statistiques sont mobilisés : $\mathrm{R}^{2}$, GoF, AVE.

Tableau 6 : Résultats de validation du modèle structurel

\begin{tabular}{|l|l|l|l|}
\hline GoF & GoF (Bootstrap) & $\mathrm{R}^{2}-F(93.107)$ Pr $>F: 0,000$ & AVE \\
\hline Absolute $=0.640$ & 0.639 & \multirow{2}{*}{0.640 (bootstrap) } & \\
\cline { 1 - 2 } Relative $=0.954$ & 0.932 & 0.631 ( $\mathrm{R}^{2}$ ajusté $)$ & 0.644 \\
\cline { 1 - 2 } External model $=0.998$ & 0.994 & & \\
\hline Internal model $=0.956$ & 0.938 & \\
\hline
\end{tabular}

N.B: Bootstrap - 300 re-sampling

Le modèle est validé car les indicateurs statistiques dépassent les seuils recommandés.

L'expression du modèle structurel est présentée dans le tableau ci-dessous.

Tableau 7: Path coefficients (XLSTAT PLSPM software)

\begin{tabular}{|l|c|c|c|c|c|}
\hline & $\begin{array}{c}\text { Satisfaction } \\
\text { point de retrait }\end{array}$ & $\begin{array}{c}\text { Satisfaction } \\
\text { service }\end{array}$ & $\begin{array}{c}\text { Satisfaction } \\
\text { site internet }\end{array}$ & $\begin{array}{c}\text { Satisfaction } \\
\text { produits }\end{array}$ & Freins \\
\hline $\begin{array}{l}\text { Path } \\
\text { coefficient }\end{array}$ & 0.371 & 0.166 & 0.223 & 0.151 & -0.061 \\
\hline
\end{tabular}

Ce modèle nous permet de déterminer les variables créatrices de valeur et leurs poids respectifs : Comportement de recommandation $=0.371$ satisfaction point de retrait +0.223 satisfaction site internet +0.166 satisfaction service +0.151 satisfaction produits -0.061 freins.

Nous remarquons que deux variables ressortent de manière significative pour expliquer le comportement de recommandation d'un Drive (59,1\% pour ces deux variables) :

- la satisfaction du point de retrait contribuant à 37,1\% de la valeur perçue par le client,

- la satisfaction du site internet expliquant 22,3\% de la valeur perçue par le client.

\footnotetext{
${ }^{8}$ Les analyses menées en vue de valider le modèle structurel sont là encore réalisées à l'aide du logiciel Xlstat PLSPM.

${ }^{9}$ Le poids de chaque variable explicative est illustré par la valeur du chemin causal (path-coefficient).
} 
Plus précisément, dans sa recommandation le client va privilégier tout d'abord et de manière significative «le point de retrait». Le consommateur est donc sensible à l'ensemble des critères constituant le Drive physique: temps d'attente, facilité d'accès routier, facilité d'authentification et relation avec le personnel. Il est à noter que la notation accordée pour chacun de ces critères se révèle très proche : temps d'attente $(8,37)$; facilité $d$ 'accès routier $(8,55)$; facilité d'authentification $(8,63)$; relation avec le personnel $(8,70)$.

Le poids important accordé à cette variable conforte l'importance du facteur temps : le Drive doit être accessible et sources de gains de temps des activités jugées sans valeur ajoutée car routinières. Ensuite, ce positionnement met en exergue la sensibilité du consommateur au critère « relation clientèle » et par conséquent l'importance accordée au personnel de contact dans la relation de service.

Nous constatons que deux critères semblent moins prépondérants dans le comportement de recommandation du Drive :

- la satisfaction du service reçu formant $16,6 \%$ de la valeur client ;

- la satisfaction de la commande de produits contribuant à 15,1\% de la valeur délivrée.

Au regard de leur classement, ces deux variables ont moins d'importance aux yeux du client car pour celui-ci, elles ne sont pas des variables sources de différenciation. Dès lors, elles apparaissent comme des variables que toute enseigne se doit de proposer a minima. Plus précisément, le positionnement du critère «services »souligne en particulier le fait que les distributeurs sont dans l'obligation de garantir la disponibilité du produit, au bon endroit (l'importance de l'emplacement) et au bon moment (l'importance du temps) car le consommateur choisit cette formule de service pour s'affranchir d'une ensemble de tâches sans valeur ajoutée.

Enfin, afin d'affiner ce modèle, nous l'avons testé sur les trois types de drive (déporté, accolé et picking magasin).

Le troisième résultat souligne donc des différences entre le modèle causal général et les différents types de Drive ( $c f$. tableau 9).

Tableau 8 : Résultats de validation des modèles structurels par type de drive

\begin{tabular}{|l|c|c|c|}
\hline & DEPORTE & ACCOLE & PICKING MAGASIN \\
\hline $\mathrm{R}^{2}$ (Bootstrap - 300) & 0.667 & 0.687 & 0.801 \\
\hline Average of variance extracted & 0.673 & 0.655 & 0.515 \\
\hline $\begin{array}{l}\text { Goodness of Fit after } \\
\text { bootstrap (absolute and } \\
\text { relative) }\end{array}$ & 0.669 et 0.928 & 0.655 et 0.857 & 0.625 et 0.843 \\
\hline
\end{tabular}


Les techniques des tests multi-groupes et de permutation sont mobilisées (avec PLSPM). Les résultats du tableau ci-dessus permettent de valider les trois modèles structurels.

Tableau 9 : Les variables créatrices de valeur par type de Drive

\begin{tabular}{|l|l|}
\hline Drive déporté & $\begin{array}{l}0.338 \text { satisfaction point de retrait }+0.270 \text { satisfaction site internet }+0.190 \text { satisfaction } \\
\text { service }+0.128 \text { satisfaction produits }-0.051 \text { freins }\end{array}$ \\
\hline Drive accolé & $\begin{array}{l}0.510 \text { satisfaction point de retrait }+0.235 \text { satisfaction produits }+0.084 \text { satisfaction } \\
\text { service }+0.010 \text { satisfaction site internet }+0.009 \text { freins }\end{array}$ \\
\hline Drive picking magasin & $\begin{array}{l}0.443 \text { satisfaction point de retrait }+0.435 \text { satisfaction site internet }+0.013 \text { satisfaction } \\
\text { service }+0.168 \text { satisfaction produits }-0.067 \text { freins }\end{array}$ \\
\hline
\end{tabular}

Ainsi, nous constatons un résultat constant à tous les modèles : la satisfaction du point de retrait est systématiquement la variable créatrice de valeur pour le client quel que soit le type de Drive. Plus précisément, quel que soit le modèle de Drive, le consommateur exige que celui-ci répondre à l'ensemble des critères constituant le Drive physique : temps d'attente, facilité d'accès routier, facilité d'authentification et relation avec le personnel.

Ensuite, le Drive de type accolé est le seul à proposer un modèle de création de valeur totale ; autrement dit, les freins rencontrés par le client ne détruisent pas sa perception générale du Drive et n'induisent pas de comportement négatif. Nous pouvons donc avancer que le modèle le plus performant - créateur de valeur - du point de vue du client semble être le Drive accolé. Plus précisément, nous notons que pour ce modèle de Drive la satisfaction du point de retrait contribue pour $51 \%$ de la valeur délivrée, suivie de la satisfaction produit $(23,5 \%$ de la valeur délivrée). Par contre la satisfaction «services » et la satisfaction du site internet, respectivement $8,4 \%$ et $10 \%$ de la valeur, apparaissent bien moins importants comme variables créatrices de valeur. Nous pouvons aussi avancer que la variable «satisfaction des produits » ne contribue que pour $23,5 \%$ de la valeur car si le client utilise le Drive pour effectuer ces achats répétitifs il a aussi la possibilité de réaliser des achats complémentaires dans le magasin du Drive accolé.

Par ailleurs, nous pouvons noter que les facteurs clés de succès du Drive de type déporté semblent finalement être les mêmes que ceux qui structurent le modèle général. Enfin, le Drive de type picking magasin se distingue des autres modèles. En effet, la valeur créée aux yeux des clients s'explique principalement par deux variables, la satisfaction du point de retrait (pour 44,3\%) et la satisfaction du site internet (pour 43,5\%). Par contre la satisfaction «produits» et la satisfaction «service» ont peu d'importance dans la valeur créée. L'ensemble de ces résultats pour ce modèle particulier de Drive s'explique par le fait que ce 
type de Drive, très dépendant d'un magasin physique, est capable de proposer un nombre de références bien plus important que dans le cas des deux autres modèles. Avec ce type de modèle, le client est susceptible de réaliser à la fois ces achats «corvées » et répétitifs tout en profitant de la proximité du magasin pour réaliser ces achats complémentaires. Aussi, le client sera essentiellement sensible aux éléments déjà évoqués dans le modèle général : temps d'attente, facilité d'accès routier, facilité d'authentification et relation avec le personnel.

\section{2-3- Implications managériales}

Au regard de nos résultats, la proximité géographique s'avère une préoccupation majeure des consommateurs. Elle confirme tout l'intérêt qu'il y a pour les distributeurs d'envisager le Drive comme une formule de service spatiale, presque «hors sol» comparé aux hypermarchés traditionnels, leur permettant de parfaitement coller aux attentes actuelles et aux besoins en termes de gain de temps d'une typologie spécifique de consommateurs, à savoir aujourd'hui les catégories socio-professionnelles intermédiaires.

En ce sens, le géomarketing va s'installer comme un domaine informationnel prépondérant en Drive quand on sait que 50,9\% de la valeur ajoutée du modèle est généré par un positionnement spatial à proximité du domicile des clients et $26,7 \%$ par la proximité du lieu de travail. Au total, c'est plus de $75 \%$ de la valeur ajoutée des Drive qui s'inscrit sous dépendance du géomarketing et donc de l'art pour une enseigne de savoir positionner ses Drive dans une logique essentiellement économico-spatiale. Par conséquent, la localisation des drive représente un avantage concurrentiel autonome et spécifique immédiat en termes de fréquentation et le choix du drive par l'utilisateur ainsi qu'un levier déterminant dans la stratégie d'optimisation du maillage territorial.

Ensuite, si le consommateur exige, quel que soit le modèle de Drive, que celui-ci soit source de gains de temps car facilement accessible, il n'en demeure pas moins que ce dernier reste aussi très sensible à la qualité de la relation établie avec le personnel de contact au sein de cette nouvelle formule de service. Les enseignes doivent donc être bien conscientes que l'accueil du client par la personne chargée de la livraison au Drive est aussi un moment essentiel dans la consolidation de la relation de service. Ce face à face est donc susceptible d'enrichir la relation de service et offre la possibilité d'assurer le développement du capitale- 
marque de l'enseigne d'autant plus que le consommateur a tendance à rester fidèle au magasin qu'il fréquente habituellement ${ }^{10}$.

Une telle fidélité à leur enseigne souligne le fait que le client perçoit le Drive comme un service complémentaire au regard des différentes formules proposées par le distributeur. Dans ce cas le Drive a bien pour vocation consolider la relation de service établie par l'enseigne avec le consommateur. Nous pouvons souligner le fait que le Drive apparait bien plus captif que les formules traditionnelles pour lesquelles les clients multiplient les alternatives entre un magasin principal, un secondaire et des occasionnels. En définitive, en GMS, les services s'inscrivent progressivement comme des activités commerciales pourvoyeuses de marges dans un univers où l'offre alimentaire devient un moyen de générer des marges additionnelles en service (figure $\mathrm{n}^{\circ} 2$ ).

Figure $n^{\circ} 2$ : L’explosion des services dans la grande distribution alimentaire

\section{Evolution des facteurs distinctifs des services en distribution alimentaire}

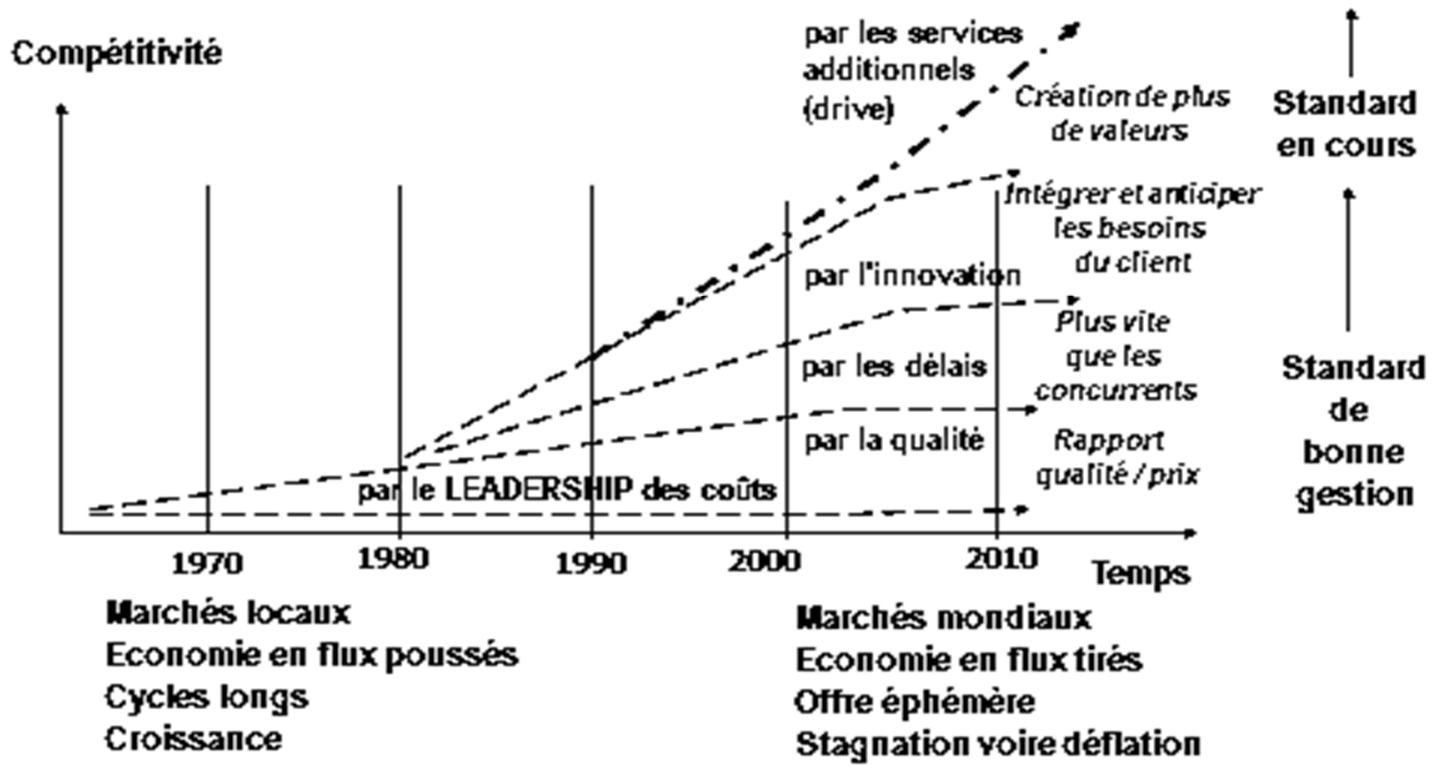

En effet, si la grande distribution s'est construite par le leadership des coûts, elle s'est développée par la qualité, les délais, l'innovation dans ses processus et aujourd'hui par les services additionnels dont le plus remarquable est le Drive. Aujourd'hui, ce sont donc les

\footnotetext{
${ }^{10}$ Dans l'enquête que nous avons menée, près de $70 \%$ des consommateurs fréquentent le drive de leur magasin habituel, contre $19 \%$ le Drive proche de leur lieu de travail et $18 \%$ le drive d'un magasin proche de leur domicile.
} 
services additionnels qui apparaissent susceptible de créer le plus de valeur. Aussi, pour se prémunir à terme d'une possible cannibalisation des canaux entre eux, les GSA doivent être attentives à développer une offre de services cohérente afin d'éviter tout chevauchement entre les différentes formules de service proposées au client.

La pérennité du Drive dépend donc du respect des trois règles d'or qui ont, jusqu'à présent, toujours rythmés la gestion opérationnelle des GMS à savoir satisfaire le client par le prix et le service, optimiser au maximum les flux logistiques et, enfin, privilégier le volume sur la marge. Aussi, tout comme les hypermarchés, les supermarchés, la proximité et le harddiscount, le Drive est tributaire d'une performance, à la fois, marketing mais aussi logistique. En effet, au regard des variables créatrices de valeur de notre modèle validé, la formule Drive s'avère exigeante vis-à-vis de la mise à disposition du bon produit au bon moment avec la bonne information. Dès lors la maitrise de la logistique s'avère indispensable car elle participe à la construction du taux de service. Plus précisément, il s'agit d'élaborer des structures susceptibles d'assurer les fonctions d'un entrepôt alliant à la fois vitesse, disponibilités des produits et réactivité tout en maitrisant l'ensemble des coûts logistiques inhérents à ce type de structure. Cette maitrise de la logistique peut s'avérer encore plus prégnante si les enseignes souhaitent faire évoluer la formule du Drive vers une formule bien plus exigeante dans la gestion logistique du dernier kilomètre: la formule LAD. Pour maitriser la frontière cout/service de ce service additionnel proposé au client, les enseignes ont la possibilité de s'appuyer sur leur drive déporté dans le but d'alimenter des points de retraits, des quartiers, des clients à domicile dans une stratégie offensive

\section{Conclusion}

Au regard d'un environnement connaissant de profonds changements la formule de service du Drive a pour vocation de consolider la relation de service établie par l'enseigne à son client. Plus précisément, il ressort de l'enquête que le client recherche principalement en termes de valeur un point de retrait capable de le satisfaire dans sa recherche de gains de temps. La proximité semble jouer un rôle majeur dans cette relation de service novatrice. Ensuite, pour que l'ensemble de la prestation de service se déroule de manière fluide et sans accroche le client exige que le distributeur soit dans la capacité à maitriser une logique de flux tendu (une commande dans une plage horaire prédéfini) : la performance logistique doit être continuellement garantie par le distributeur. Enfin, si le Drive solo reste structurellement dominé en termes de création de valeur 
par le Drive accolé, la création d'un pôle commerce autour du Drive solo semble de nature à inverser le rapport de force. En effet, le succès d'un Drive semble, à la fois, dépendre d'un effet réseau d'enseigne mais aussi d'un fort effet lié au pôle de chalandise considéré. Il est donc possible d'envisager différemment la structuration d'un Drive solo autour d'un pôle renforcé de commerces de proximité (services automobiles, magasins bio, cafétéria haut de gamme, magasins de producteurs,) bénéficiant d'une grande facilité d'accès au point de retrait. 


\section{Bibliographie}

Anquez M.C., (2004), Elles font les courses au pas de course, in Temps des courses, courses des temps sous la direction de Bondue J.P., Publication de l'Université de Lille 1, pp. 109118.

Baranger P., Dang N'Guyen G.; Leray Y.; Mevel O., (2009), Le Management Opérationnel des Services, Techniques de Gestion, Economica.

Bouzid Y., Vanheems R., (2014), Comportement web to store : vers une nouvelle logique de contrôle de soi ? Management \& Avenir, nº71, pp. 189-200.

Chin W. W. (1998), Issue and opinion on structural equation., MIS Quarterly, pp. 7-16.

Churchill G.A. (1979), A paradigm for developing better measures of marketing constructs, Journal of Marketing Research, 16, pp. 64-73

Cliquet G. (2002), Le géomarketing: méthodes et stratégie du marketing spatial, in Le géomarketing : méthodes et stratégies du marketing spatial, éd Cliquet G., Hermès.

Colla E. et Lapoule P. (2015), Le drive : vecteur de cannibalisation ou de complémentarité ? Le cas de la grande distribution alimentaire, Revue Française du Marketing, n²52, pp. 55-70.

Colla E. et Lapoule P. (2012), E-commerce: exploring the critical success factors, International Journal of Retail and Distribution Management, vol 40, n ${ }^{\circ} 1$, pp.842-864.

Colla E., Lapoule P., (2011), Les facteurs-clés du succès des cybermarchés : les enseignements du cas Tesco, Décision Marketing, vol 61, pp. 35-45.

Eiglier P., Langeard L., (1987), Servuction : le marketing des services, McGraw-Hill.

Gadrey J. (1992), L'économie des services, Editions de la Découverte.

Gahinet M.C., (2014), les nouveaux formats alimentaires de proximité : regards croisés des distributeurs et des consommateurs, Management et Avenir, pp.153-168.

Guillo P.A. (2008), Les stratégies d'expansion dans la grande distribution : le cas des maxidiscompteurs en France, Décisions Marketing, n50, pp. 7-18.

Heitz M., Douard J.P., Cliquet G. (2011), Grande distribution alimentaire et drive: une solution à la mobilité des consommateurs, Congrès E. Thil, Roubaix.

Huré E., Cliquet G., (2011), From a multi- to a cross-channel shopping experience in grocery retail environment, in EIRASS Conference, San Diego, États-Unis.

Huré E., Vo T., Cliquet G., Durand B., (2013), E-supply chain et productivité : le cas de la distribution alimentaire, Revue Française de Gestion Industrielle, vol 32, n 1 , pp 25-45

Lovelock Ch., Wirtz J., Lapert D., Munos A., (2011), Marketing des services, 7ème edition, Pearson.

Mevel O., Morvan Th., (2015), Drive, entropie et logistique urbaine : qu'attendre de la nouvelle relation de service initiée par les GMS avec les consommateurs ? Logistique \& Management, vol 23, n², pp. 21-30.

Marouseau G., (2013), Le click and Collect: la logistique participative du client dans les Drive, Logistique \& Management, vol 21, n³, pp. 31-39

Picot-Coupey K., Cliquet G., Petr Ch., (2009), hypermarché, cybermarché et courses alimentaires : quels projets d'usage par les consommateurs? 9th International Conférence Marketing Trend, Venice 
Punakivi M., Yrjölä H., Holmström J., (2001), Solving the last mille issue: reception box and delivery box, International Journal of Physical distribution and Logistics Management, vol $31, \mathrm{n}^{\circ} 6, \mathrm{pp} .427-439$.

Rouquet A., (2014), La distribution par "drive" : définition et typologie, Décision Marketing, $\mathrm{n}^{\circ} 75$, juillet-septembre, pp. 65-78.

Rouquet A., Goudarzi K., Henriquez T., (2010), Le transfert d'activités logistiques entrepriseconsommateur : une comparaison des cas Auchan Drive et IKEA, Logistique \& Management, vol 18, n², pp. 49-61.

Samii A.K., (2004), Stratégie Logistique, Dunod.

Shapiro R.D, Heskett J.L., (1985), Logistics strategy cases and concept, West publishing Co.

Tenenhaus M., Vinzi V.E., Chatelin Y-M. et Lauro C. (2005), PLS Path modelling, Computational Statistics and Data Analysis, n)48, pp. 159-205.

Torre A. (2014). Relation de proximité et comportements d'innovation des entreprises des clusters, Revue Française de Gestion, n 242 , pp. 49-80.

Volle M. (2000), E-économie, Economica, Paris 
Annexe 1 : Le modèle causal général

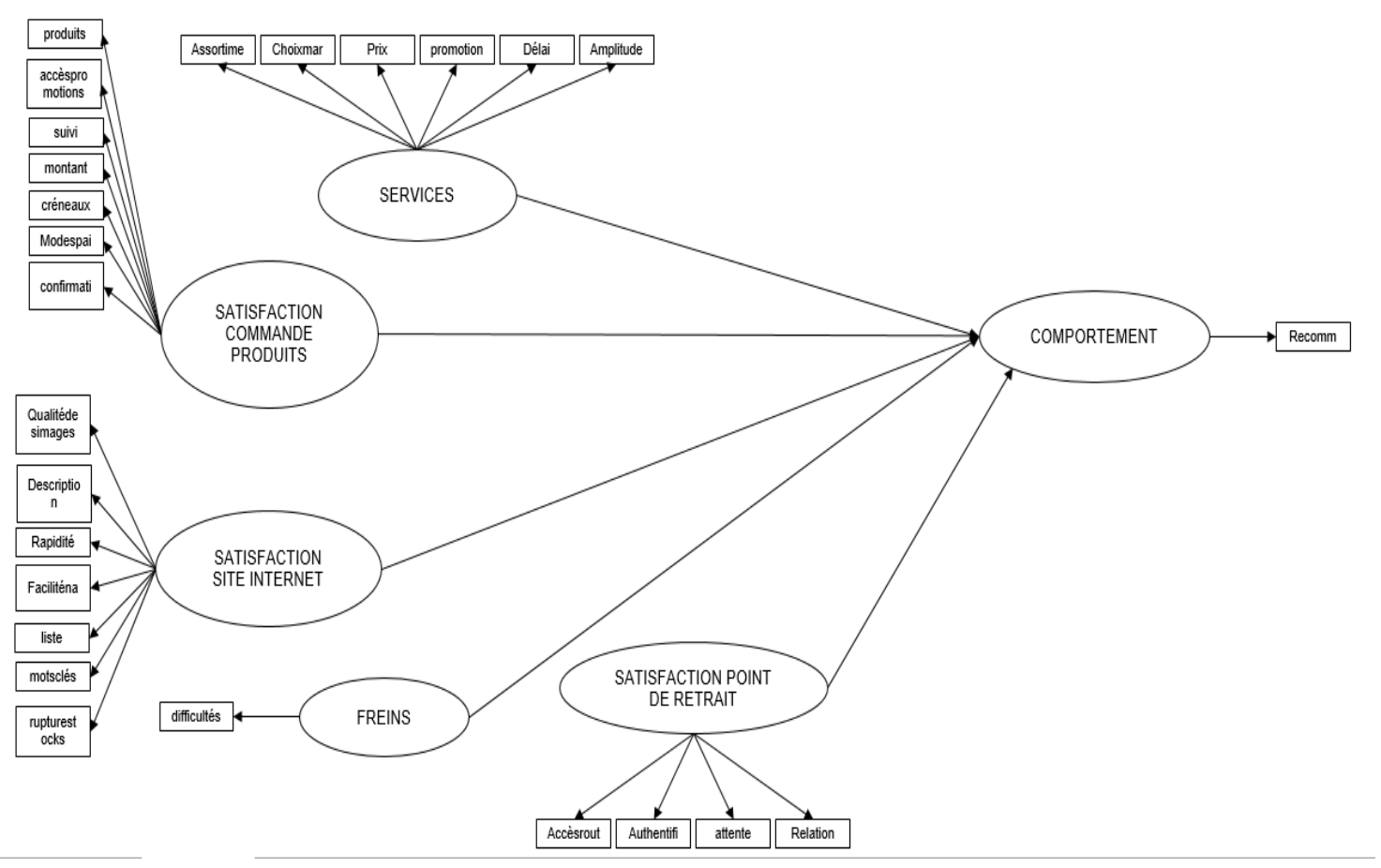

Annexe 2 : Classement des critères de choix d'un Drive ${ }^{11}$

\begin{tabular}{|c|c|c|c|c|c|c|c|c|}
\hline Position & $\begin{array}{l}\text { Proximité de } \\
\text { mon domicile } \\
\quad(\text { en } \%)\end{array}$ & $\begin{array}{c}\text { Proximité de } \\
\text { mon lieu de } \\
\text { travail (en\%) }\end{array}$ & $\begin{array}{c}\text { Sur le trajet entre } \\
\text { mon domicile et } \\
\text { mon travail (en\%) }\end{array}$ & $\begin{array}{l}\text { Choix des } \\
\text { produits } \\
\text { (en\%) }\end{array}$ & $\begin{array}{c}\text { Prix } \\
\text { pratiqués } \\
(\text { en\%) }\end{array}$ & $\begin{array}{c}\text { Choix des } \\
\text { marques } \\
(\text { en } \%)\end{array}$ & $\begin{array}{c}\text { Facilité } \\
\text { d'accès au } \\
\text { point de } \\
\text { retrait }(\mathbf{e n} \%) \\
\end{array}$ & $\begin{array}{c}\text { L'ergonomie } \\
\text { du site de } \\
\text { commande } \\
(\text { en } \%) \\
\end{array}$ \\
\hline 1 & 50,9 & 8,7 & 8,3 & 7,6 & 8,1 & 1,1 & 12,8 & 2,4 \\
\hline 2 & 13,5 & 26,7 & 9,1 & 12,6 & 11,3 & 1,7 & 14,3 & 6,3 \\
\hline 3 & 7,6 & 5,2 & 22,4 & 11,5 & 9,6 & 6,3 & 11,5 & 7,6 \\
\hline 4 & 2,8 & 1,9 & 4,8 & 18,5 & 11,1 & 6,7 & 9,8 & 5,9 \\
\hline 5 & 2,4 & 3,1 & 1,3 & 7,0 & 18,1 & 11,5 & 9,4 & 5,9 \\
\hline 6 & 2,6 & 2,6 & 4,3 & 5,7 & 5,4 & 21,1 & 7,2 & 8,9 \\
\hline $\begin{array}{c}\text { Non } \\
\text { Classé }\end{array}$ & 20,2 & 51,9 & 49,8 & 37,0 & 36,3 & 51,7 & 35,0 & 59,8 \\
\hline Total & 100,0 & 100,0 & 100,0 & 100,0 & 100,0 & 100,0 & 100,0 & 100,0 \\
\hline
\end{tabular}

${ }^{11}$ Dans l'analyse de ces résultats nous tenons compte des valeurs les plus élevées et du nombre de citation (ex : proximité du lieu de travail est classé par $26,7 \%$ des clients en 2e position mais il n'a été classé que par $48 \%$ des sondés, tandis que "le choix des produits" classé en 2e position par 12,6\% des clients a été cité par 63\% des sondés) 
Annexe 3 : Matrice de validité discriminante des construits du modèle

\begin{tabular}{|l|l|l|l|l|l|l|l|}
\hline & $\begin{array}{l}\text { SATISFAC } \\
\text { TION } \\
\text { PRODUIS }\end{array}$ & $\begin{array}{c}\text { SATISFAC } \\
\text { TINN SITE } \\
\text { INTERNET }\end{array}$ & FREINS & $\begin{array}{c}\text { SATISFAC } \\
\text { TION } \\
\text { SERVICE }\end{array}$ & $\begin{array}{c}\text { SATISFACTION } \\
\text { POINT DE } \\
\text { RETRAIT }\end{array}$ & $\begin{array}{c}\text { COMPORTE- } \\
\text { MENT }\end{array}$ & $\begin{array}{c}\text { Moyenne } \\
\text { Communalit } \\
\text { és (AVE) }\end{array}$ \\
\hline $\begin{array}{l}\text { SATISFACTION } \\
\text { PRODUITS }\end{array}$ & $\mathbf{1}$ & 0.524 & 0.011 & 0.546 & 0.454 & 0.461 & 0.587 \\
\hline $\begin{array}{l}\text { SATISFACTION SITE } \\
\text { INTERNET }\end{array}$ & 0.524 & $\mathbf{1}$ & 0.033 & 0.525 & 0.433 & 0.470 & 0.644 \\
\hline FREINS & 0.011 & 0.033 & $\mathbf{1}$ & 0.001 & 0.010 & 0.001 & \\
\hline $\begin{array}{l}\text { SATISFACTION } \\
\text { SERVICE }\end{array}$ & 0.546 & 0.525 & 0.001 & $\mathbf{1}$ & 0.459 & 0.474 & 0.631 \\
\hline $\begin{array}{l}\text { SATISFACTION } \\
\text { POINT DE RETRAIT }\end{array}$ & 0.454 & 0.433 & 0.010 & 0.459 & $\mathbf{1}$ & 0.526 & 0.765 \\
\hline COMPORTEMENT & 0.461 & 0.470 & 0.001 & 0.474 & 0.526 & $\mathbf{1}$ & \\
\hline $\begin{array}{l}\text { Moyenne } \\
\text { Communalités (AVE) }\end{array}$ & 0.587 & 0.644 & & 0.631 & 0.765 & & $\mathbf{0}$ \\
\hline
\end{tabular}

Annexe 4 : Les caractéristiques de l'échantillon (enquête réalisée de mars à mai 2015)

\begin{tabular}{|l|c|}
\hline Age & Pourcentage \\
\hline $18-29$ ans & 13,4 \\
\hline $30-44$ ans & $\mathbf{6 7 , 7}$ \\
\hline $45-59$ ans & 17,3 \\
\hline $60-74$ ans & 1,7 \\
\hline Total & 100,0 \\
\hline
\end{tabular}

\begin{tabular}{|c|c|}
\hline $\begin{array}{c}\text { Nombre de personnes } \\
\text { dans le foyer }\end{array}$ & Pourcentage \\
\hline 1 & 3,4 \\
\hline 2 & 19,3 \\
\hline 3 & 24,6 \\
\hline 4 & $\mathbf{3 8 , 1}$ \\
\hline 5 & 12,8 \\
\hline 6 et plus & 1,7 \\
\hline Total & 100,0 \\
\hline
\end{tabular}

\begin{tabular}{|l|c|}
\hline CSP & Pourcentage \\
\hline Agriculteurs exploitant & 1,30 \\
\hline $\begin{array}{l}\text { Artisan, commerçants, chefs } \\
\text { d'entreprise }\end{array}$ & 3,20 \\
\hline $\begin{array}{l}\text { Cadres et professions } \\
\text { intellectuelles supérieures }\end{array}$ & 13,20 \\
\hline Employés & $\mathbf{3 7 , 1 0}$ \\
\hline Professions intermédiaires & $\mathbf{2 6 , 3 0}$ \\
\hline Ouvriers $\quad$ Total & 13,20 \\
\hline \multicolumn{1}{|c|}{} & 100,0 \\
\hline
\end{tabular}

Annexe 5 : Les types de Drive fréquentés au sein de l'échantillon (enquête réalisée de mars à mai 2015)

\begin{tabular}{|c|c|c|c|c|c|}
\hline \multirow[b]{2}{*}{ Enseigne } & \multirow[b]{2}{*}{ Fréquentation } & \multicolumn{3}{|c|}{ Type de Drive fréquenté } & \multirow[b]{2}{*}{ Total } \\
\hline & & Déporté & Accolé & $\begin{array}{l}\text { Picking } \\
\text { magasin }\end{array}$ & \\
\hline Auchan Drive & $0,18 \%$ & $100,0 \%$ & $0,0 \%$ & $0,0 \%$ & $100,0 \%$ \\
\hline Carrefour Drive & $1,80 \%$ & $40,0 \%$ & $60,0 \%$ & $0,0 \%$ & $100,0 \%$ \\
\hline Casino & $0,36 \%$ & $0,0 \%$ & $100,0 \%$ & $0,0 \%$ & $100,0 \%$ \\
\hline ChronoDrive & $0,36 \%$ & $100,0 \%$ & $0,0 \%$ & $0,0 \%$ & $100,0 \%$ \\
\hline Cora Drive & $3,24 \%$ & $0,0 \%$ & $100,0 \%$ & $0,0 \%$ & $100,0 \%$ \\
\hline Courses U & $6,12 \%$ & $5,9 \%$ & $58,8 \%$ & $35,3 \%$ & $100,0 \%$ \\
\hline Le Drive Intermarché & $8,63 \%$ & $0,0 \%$ & $31,3 \%$ & $68,8 \%$ & $100,0 \%$ \\
\hline Leclerc Drive & $79,32 \%$ & $59,5 \%$ & $39,1 \%$ & $1,4 \%$ & $100,0 \%$ \\
\hline Total & & $50,4 \%$ & $40,1 \%$ & $9,5 \%$ & $100,0 \%$ \\
\hline
\end{tabular}

